\title{
Volatile Fingerprinting (SPME-GC-FID) to Detect and Discriminate Diseases of Potato Tubers
}

\author{
A. C. Kushalappa and L. H. Lui, Department of Plant Science, McGill University, Ste-Anne de Bellevue, Québec, \\ Canada H9X 3V9; C. R. Chen, Food Science and Agriculture Chemistry, McGill University; and B. Lee, Agricul- \\ ture and Agri-Food Canada, Food Research and Development Center, Saint-Hyacinthe, Québec, Canada J2S 8E3
}

\begin{abstract}
Kushalappa, A. C., Lui, L. H., Chen, C. R., and Lee, B. 2002. Volatile fingerprinting (SPMEGC-FID) to detect and discriminate diseases of potato tubers. Plant Dis. 86:131-137.

Volatiles from Russet Burbank potatoes inoculated with Erwinia carotovora subsp. carotovora, E. carotovora subsp. atroseptica, Pythium ultimum, Phytophthora infestans, or Fusarium sambucinum were monitored by sampling the head space 3,4 , and 5 days after inoculation, using a solid phase microextraction (SPME) fiber to trap and gas chromatography with flame ionization detector (GC-FID) to fingerprint volatiles. Noninoculated (NON) potatoes served as the control. Volatile fingerprints varied among diseases. Within a disease, the fingerprints varied with time since inoculation and among blocks. In general, more volatiles were observed on the fourth and fifth day after inoculation than on the third day. The amount of volatile compounds produced (peak area) within a disease group increased with incubation time; however, the variation among blocks was much higher. The amount of volatiles produced, in general, was associated with disease severity. Disease-specific volatiles were observed. The $F$. sambucinum chromatogram had two unique peaks at retention time $(\mathrm{RT})=14.1$ and $17.3 \mathrm{~min}$. P. infestans produced few peaks and the profile was quite similar to NON. In contrast, E. carotovora subsp. carotovora, E. carotovora subsp. atroseptica, and Pythium ultimum produced many peaks, and the P. ultimum was different from the bacteria, in that the chromatogram peaks at RT $=4.04$ and 8.76 min were absent. Instead, it produced a distinct peak at RT $=1.71 \mathrm{~min}$. E. carotovora subsp. carotovora and E. carotovora subsp. atroseptica couldn't be discriminated based on unique peaks; however, they varied in concentration of volatiles produced. E. carotovora subsp. carotovora produced more of $\mathrm{RT}=2.0 \mathrm{~min}$ and less of $\mathrm{RT}=2.3$ and $2.44 \mathrm{~min}$ than $E$. carotovora subsp. atroseptica. A back-propagation network (using neural networks) was developed to classify volatile profiles into six disease-groups. Cross-validation classification probabilities were $\mathrm{NON}=71$, E. carotovora subsp. carotovora $=71$, E. carotovora subsp. atroseptica $=71$, P. ultimum $=67$, Phytophthora infestans $=46$, and $F$. sambucinum $=75 \%$.
\end{abstract}

Additional keywords: disease detection, disease monitoring, electronic nose, neural network, postharvest pathology

Potatoes are often stored for up to 1 year in bulk or in pallet boxes. Diseases are the most limiting factors in storage. There are several diseases which can cause loss in storage (19). Some of the major diseases are soft rot (Erwinia carotovora subsp. carotovora), soft rot and blackleg (E. carotovora subsp. atroseptica), ring rot (Clavibacter michiganensis subsp. sepedonicus), dry rot (Fusarium sambucinum), late blight (Phytophthora infestans), pink rot (Phytophthora erythroseptica), and leak (Pythium ultimum). Generally, infection occurs before or at the time of harvesting. The source of inoculum may be the plant, soil, or infected tubers. Wounding at harvest, tuber surface wetness, and high temper-

Corresponding author: A. C. Kushalappa E-mail: ajjamada.kushalappa@mcgill.ca

Accepted for publication 24 September 2001.

Publication no. D-2001-1204-03R

(C) 2002 The American Phytopathological Society ature conditions promote infection. Although severely diseased potatoes are culled at harvest, many infections pass through inspection, especially the new infections occurring at harvest. From these infections, decay lesions develop in storage and cause serious loss of tuber quality, especially if the tubers are wet during early curing period. Thus, the disease incidence of potato in storage is variable among years and growers, depending on weather conditions and production practices. Storage managers are in need of tools to assess the quality of tubers they receive for storage and, also, to monitor disease progress during storage.

Fungicides (e.g., Thiabendazole, Benomyl, and others) are applied to manage certain fungal diseases in storage (19). However, the most common method of disease control in storage is by the management of physical environment, mainly lower temperatures. After harvest, potatoes are stored for a couple of weeks at 12 to $15^{\circ} \mathrm{C}$ to speed up wound healing. The temperature is then reduced to $12^{\circ} \mathrm{C}$ for chip- ping potatoes, 8 to $10^{\circ} \mathrm{C}$ for table potatoes, and 4 to $5^{\circ} \mathrm{C}$ for seed potatoes. Forced air circulation is used to prevent condensation on tubers (2). Storage managers also depend on the smell produced by rotting tubers to detect onset of diseases. Thus, it would be interesting to know the identity of the malodorants involved, especially using simple analytical methods involving solid phase microextraction (SPME) fibers to extract and gas chromatography with flame ionization detector (GC-FID) to establish odor fingerprints. Serological methods have been used to determine inoculum or disease levels (4). Tubers were stored at $25^{\circ} \mathrm{C}$ for 6 weeks for lesion development and then plated on media to assess natural dry rot incidence (22). Soil adhering to tubers has been used to assess dry rot, but the presence of Fusarium spp. in soil may not result in tuber dry rot in all climatic conditions. Disease-specific volatiles have been found in potato $(5,16,23-$ 26) and can be used for early detection and assessment of disease incidence.

Fruits and vegetables naturally produce many volatiles during storage. Generally, the volatile production increases with disease, frost, or physical damage $(16,23,25,26)$. Fungal and bacterial pathogens attack plants by production of specific enzymes and toxins (23). The type and amount of enzymes produced vary with the pathogen, the environment, and the host substrate. Soft rot pathogens produce enzymes that macerate the cell walls and render the tissue soft and watery. The rotting tissue releases volatiles into the air, which explains why odor sniffing by experts is commonly used to detect disease in storage. Efforts have been made to detect volatiles using GC, GC and mass spectrometry (GC-MS), and, more recently, electronic nose (e-nose) $(8,20)$. Diseasespecific volatiles have been detected in the headspace of a commercial potato storage that had 10 to $20 \%$ diseased tubers (24). The concentration of volatile compounds detected in commercial storage is usually quite low due to dilution by ventilation with fresh air; therefore, techniques have been developed to preconcentrate volatiles in tube traps containing adsorbants $(13,24)$.

Diseased potatoes have been shown to produce a number of low-molecular-weight compounds, including dimethyl disulfide, 2-butanone, pentane, furan ester, styrene, and phorone $(5,12,16,24)$. However, the 
evidence of disease-specific volatiles has been quite inconsistent within and among studies. This inconsistency may be explained by variations in host, pathogen, and environmental conditions involved in pathogenicity. Multivariate statistical analysis and neural networks (NNs) have been used to solve real-life problems with complex relationships $(3,11,14)$. Networks have been used to lean patterns and to classify individuals into predefined groups. The dynamics of disease related volatile production is also complex; therefore, the use of NNs could be a potential alternative to develop a network to classify potato diseases.

Storage managers are in need of tools not only for online monitoring of diseases during storage, but also to assess the disease potential of tubers they receive for storage. There is potential to use the volatile technology under commercial conditions to assess the disease potential of tubers received for storage and, possibly, to identify diseases. The use of tube traps is time consuming and also requires special equipment for desorption of volatiles. On the other hand, the use of SPME fibers to trap volatiles may be cheap and it takes less time (14). SPME has been used to detect trace amounts of volatiles in headspace and to identify various compounds using GC/MS (14,21). Accordingly, the objective of this study was to evaluate the potential use of SPME and GC-FID to detect and discriminate diseases of potato in small samples.

\section{MATERIALS AND METHODS}

Tuber infection establishment and volatile accumulation. Potatoes, cv. Russet Burbank (grown in Canada and the United States), were obtained from retail stores, surface sterilized with $0.5 \%$ sodium hypochlorite for $15 \mathrm{~min}$, washed in sterile water, and dried under a laminar flow. In each tuber of about $200 \mathrm{~g}$, seven holes 3 $\mathrm{mm}$ in diameter, $3 \mathrm{~mm}$ deep, and about 2 $\mathrm{cm}$ apart were made with a cork borer.

Cultures of E. carotovora subsp. carotovora (isolate 1364), E. carotovora subsp. atroseptica (isolate 707), Pythium ultimum (isolate 242), Phytophthora infestans (isolate 1618), and $F$. sambucinum (isolate 2351) were obtained from the Diagnostic Center, Sainte Foy, Quebec, Canada, except for $P$. infestans, which was obtained from AAFC, Charlottetown, Prince Edward Island, Canada. The cultures of $E$. carotovora subsp. carotovora and E. carotovora subsp. atroseptica were produced in tryptic soy agar at $22^{\circ} \mathrm{C}$. Inoculum was prepared by scraping 48-h-old cultures with a needle and suspending them thereafter in sterile water. The suspensions were vortexed and adjusted to contain $10^{7}$ $\mathrm{CFU} / \mathrm{ml}$, using a spectrophotometer (18). The culture of Pythium ultimum was produced in V8 agar at $20^{\circ} \mathrm{C}$ and, after 2 weeks, a plug was transferred to V8 broth, which was incubated at room temperature on a shaker. The culture was blended and filtered through cheese cloth (1). The suspension was adjusted to $10^{4}$ sporangia/ml. The cultures of Phytophthora infestans were grown in Rye agar plates at $20^{\circ} \mathrm{C}$ and,

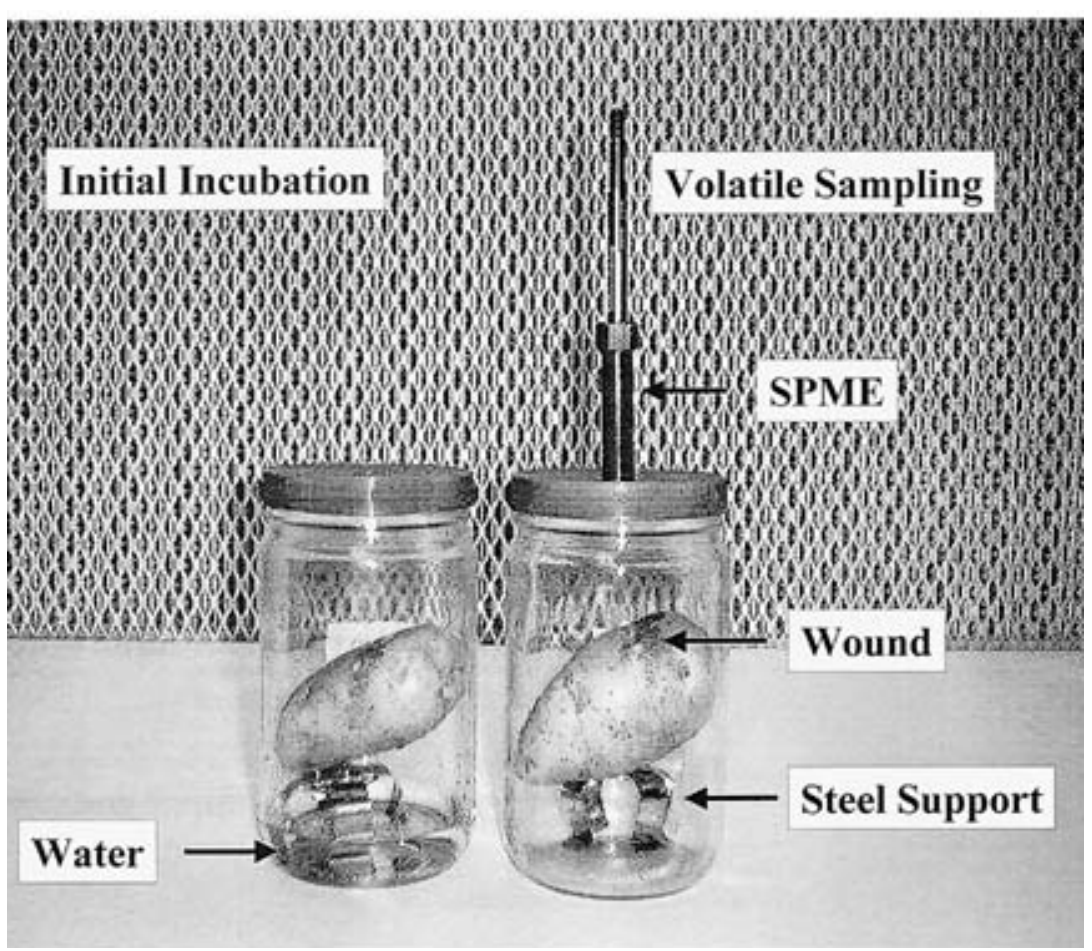

Fig. 1. Setup used for the establishment of initial infection (saturated atmosphere created with 100 $\mathrm{ml}$ of water in 1-liter septa bottle), volatile accumulation (water removed), and sampling of volatiles using solid phase microextraction (SPME) fiber. after 2 weeks, the culture was blended and the suspension was adjusted to $10^{4}$ sporangia/ml (17). The cultures of $F$. sambucinum were produced on potato dextrose agar plates at $22^{\circ} \mathrm{C}$ and a suspension of macroconidia was prepared from 7- to 10day-old cultures by flooding the plates with distilled water, vortexing the suspension, and filtering through two layers of cheese cloth. The suspension was adjusted to contain $10^{4}$ macroconidia/ml, using a hemacytometer.

One hole on each end of a tuber was inoculated with $20 \mu \mathrm{l}$ of sterile distilled water, which served as a check for any contamination by resident saprophytic microbes which can grow on moist wounded tissue, and the remaining five holes were inoculated with $20 \mu \mathrm{l}$ of the bacterial cell or fungal spore suspensions using a micropipette (10). The noninoculated (NON) control treatment consisted of all wounds in the tuber injected with sterile distilled water. The inoculated tubers were placed individually in 1-liter glass bottles containing $100 \mathrm{ml}$ of sterile distilled water at the bottom in order to provide saturated atmosphere and a thin film of free water on the surface of tubers. Stainless steel supports (two 3-cm-tall inverted $U$ strips) were used to hold the tubers above water level (Fig. 1). The bottles were sealed with caps that had silicon-based septum lining.

The tubers in bottles were incubated at $20^{\circ} \mathrm{C}$. After $24 \mathrm{~h}$, the water was removed and the bottles were resealed. The next day, the bottles were opened under a laminar flow, aerated, sealed, and then flushed with pure dry air. This procedure was repeated daily, after the volatiles were sampled. The tubers were incubated and volatiles were sampled at 3, 4, and 5 days after inoculation at $20^{\circ} \mathrm{C}$.

Volatile sampling and fingerprinting. Volatiles were sampled using an SPME fiber (\#57344-U; Supelco-Sigma-Aldrich Canada Inc., Oakville, Ontario, Canada). The SPME device consisted of a retractable fiber enclosed in a steel plunger and a holder similar to a syringe (Fig. 1). The fiber was $1 \mathrm{~cm}$ long, coated with 75$\mu$ m-thick polydimethylsiloxane/Carboxen (PDMS/Carboxen), and is recommended for low-molecular-weight organic compounds (14). The SPME fiber was conditioned at the beginning of the day of run by inserting the needle into the injection port $\left(250^{\circ} \mathrm{C}\right)$ of a gas chromatograph (Model Auto system; Perkin-Elmer, Inc., Norwalk, $\mathrm{CT}$ ), releasing the fiber, and leaving the fiber in place for one complete run. The volatiles accumulated in bottles for $24 \mathrm{~h}$ were sampled, after shaking the bottles by hand a few times, by inserting the needle through the septum and extending the fiber, exposing it to the headspace. The fiber was retrieved into the holder after $15 \mathrm{~min}$ $(14,21)$ and immediately inserted into the $\mathrm{GC}$ injection port, heated to $250^{\circ} \mathrm{C}$, for volatile analysis. The GC-FID was 
equipped with a fused silica capillary column, $30 \mathrm{~m}$ in length by $0.32 \mathrm{~mm}$ in internal diameter, with $0.2-\mu m$-thick Supelcowax film as the stationary phase (Supelcowax-10; \#24080-U; Supelco, Bellafonte, PA). The initial temperature of the oven was held at $50^{\circ} \mathrm{C}$ for $5 \mathrm{~min}$, after which it was increased at the rate of $8^{\circ} \mathrm{C} / \mathrm{min}$ until the final temperature of $160^{\circ} \mathrm{C}$ was reached. The duration of one run was $48.8 \mathrm{~min}$. The injection port temperature was $250^{\circ} \mathrm{C}$ and the FID tempera- ture was $220^{\circ} \mathrm{C}$. Helium was the carrier gas and the flow rate was $10.3 \mathrm{ml} / \mathrm{min}$ at a pressure of $175 \mathrm{kPa}$.

The data for each experiment consisted of volatile fingerprints for six disease groups (NON, E. carotovora subsp. carotovora, E. carotovora subsp. atroseptica, Pythium ultimum, Phytophthora infestans, and $F$. sambucinum) and each disease group consisted of three incubation times ( 3,4 , and 5 days). At the outset, a blank (an empty bottle with dry air) was tested and it produced no significant peak; whereas, for every block, the SPME alone was run to check for peaks. The entire experiment was conducted eight times, over a 6-month period; thus, the whole study consisted of 144 fingerprints. The data collected consisted of types of organic volatile compounds, represented by the retention time (RT) of the GC-FID output, and the concentration, measured as the relative area under the GC-FID output curve, for each significant peak detected at

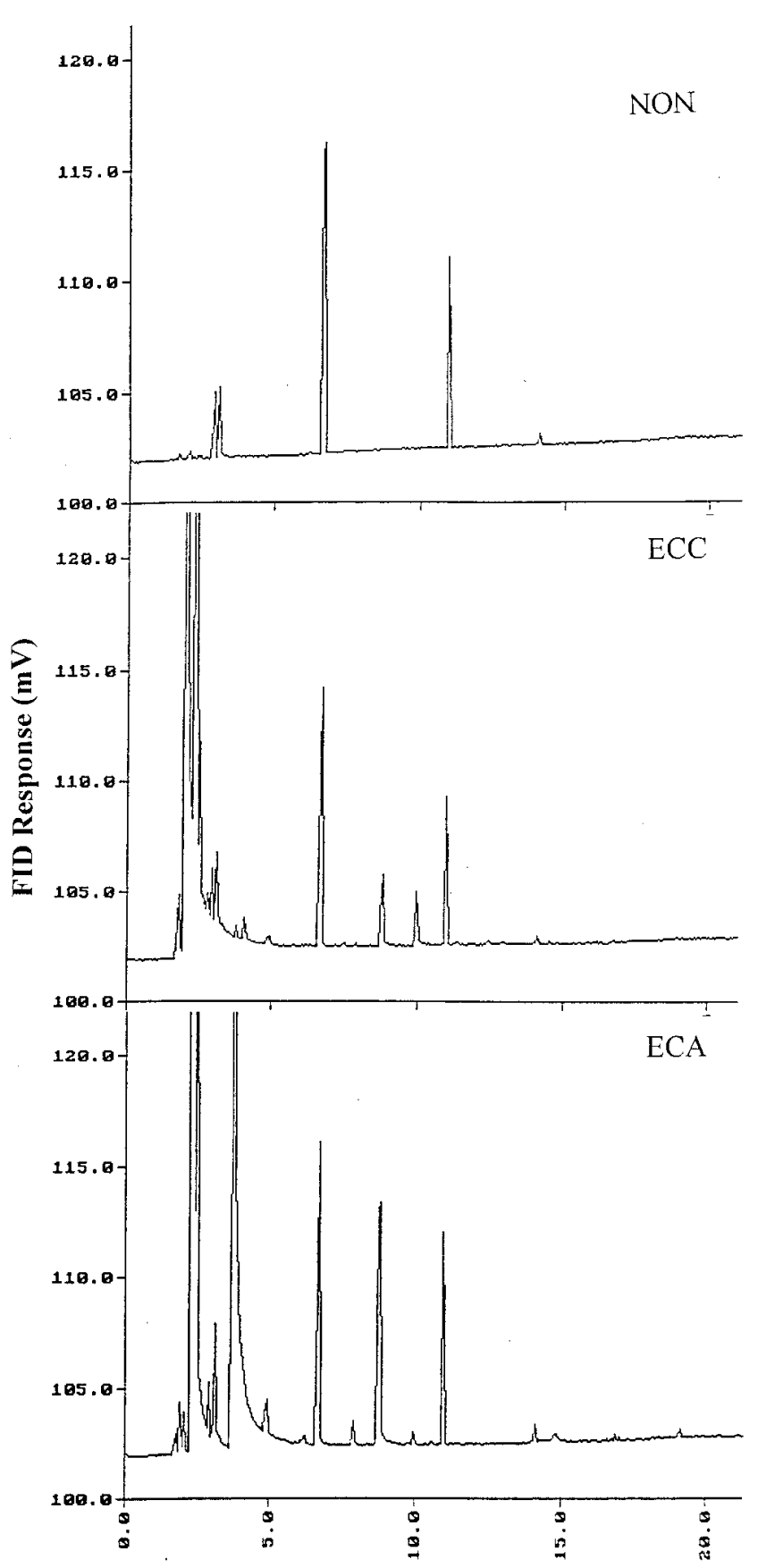

Retention Time ( $\mathrm{min})$

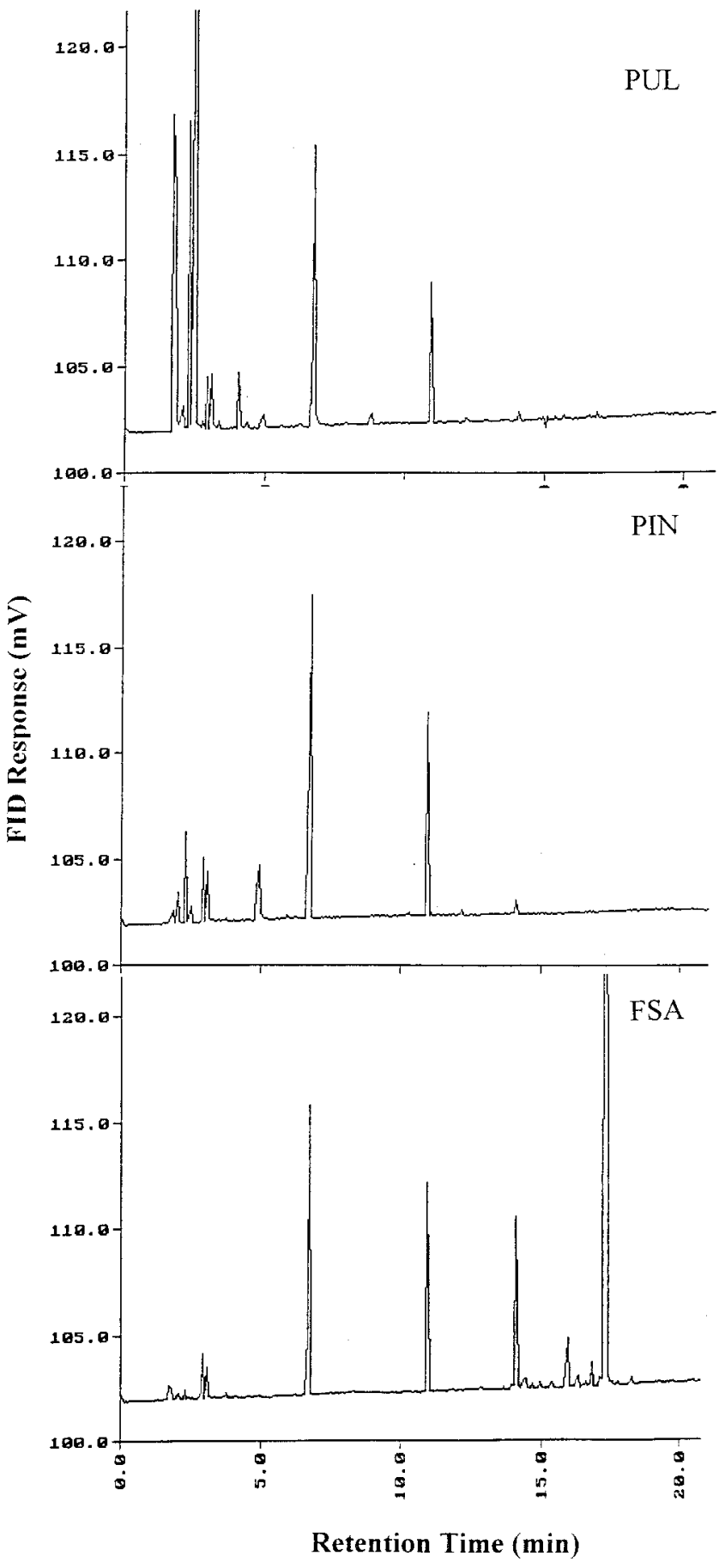

Fig. 2. Samples of volatile fingerprints for six disease-groups of potato: relative concentrations (peak area) of volatile compounds eluted at different retention times (RTs) for potato tubers noninoculated (NON), or inoculated with Erwinia carotovora subsp. carotovora (ECC), E. carotovora subsp. atroseptica (ECA), Pythium ultimum (PUL), Phytophthora infestans (PIN), or Fusarium sambucinum (FSA), using solid phase microextraction traps and a gas chromatography with flame ionization detector. Volatiles were sampled on the fifth day after inoculation; volatiles were accumulated for $24 \mathrm{~h}$. 
some RTs. The RT for a given peak varied slightly among blocks and treatments; therefore, a range of up to $0.04 \mathrm{~min}$ (average RT value given in the text) was used to include a given peak. More than 22 volatile compounds were detected, but only the 16 that were in at least four out of eight blocks in any one disease group were used for further analysis.

NN configuration. NN with the backpropagation option (15) was used to develop a potato disease network to classify volatile fingerprints into six different disease groups. The main configuration parameters to be investigated included (i) learning runs: 30,000 to 200,000; (ii) hidden layers and processing elements (PE, or neurons): 1, 2, and 3 hidden layers with 15 to 75 PEs; (iii) learning rules: Delta, normalized cumulative Delta rule (NC-Delta), and Extended Delta-Bar-Delta rule (ExtDBD); and (iv) transfer functions: hyperbolic tangent (TanH), Sigmoid, and Digital NN Architecture (DNNA). The optimal configurations of the $\mathrm{NN}$ were determined by the learning performance based on the correct classification rates for recall. The data set used for the training network consisted of 16 inputs and 6 outputs corresponding to 16 GC-FID peaks and 6 different disease groups. After training, the network was cross-validated (also known as jack-knife validation), in that the data for seven blocks were used to train the network and the remaining one block was used to test the model. Then, the test block was replaced with another block from the previous training set, and this procedure was repeated eight times. Each block consisted of 18 observations (six disease
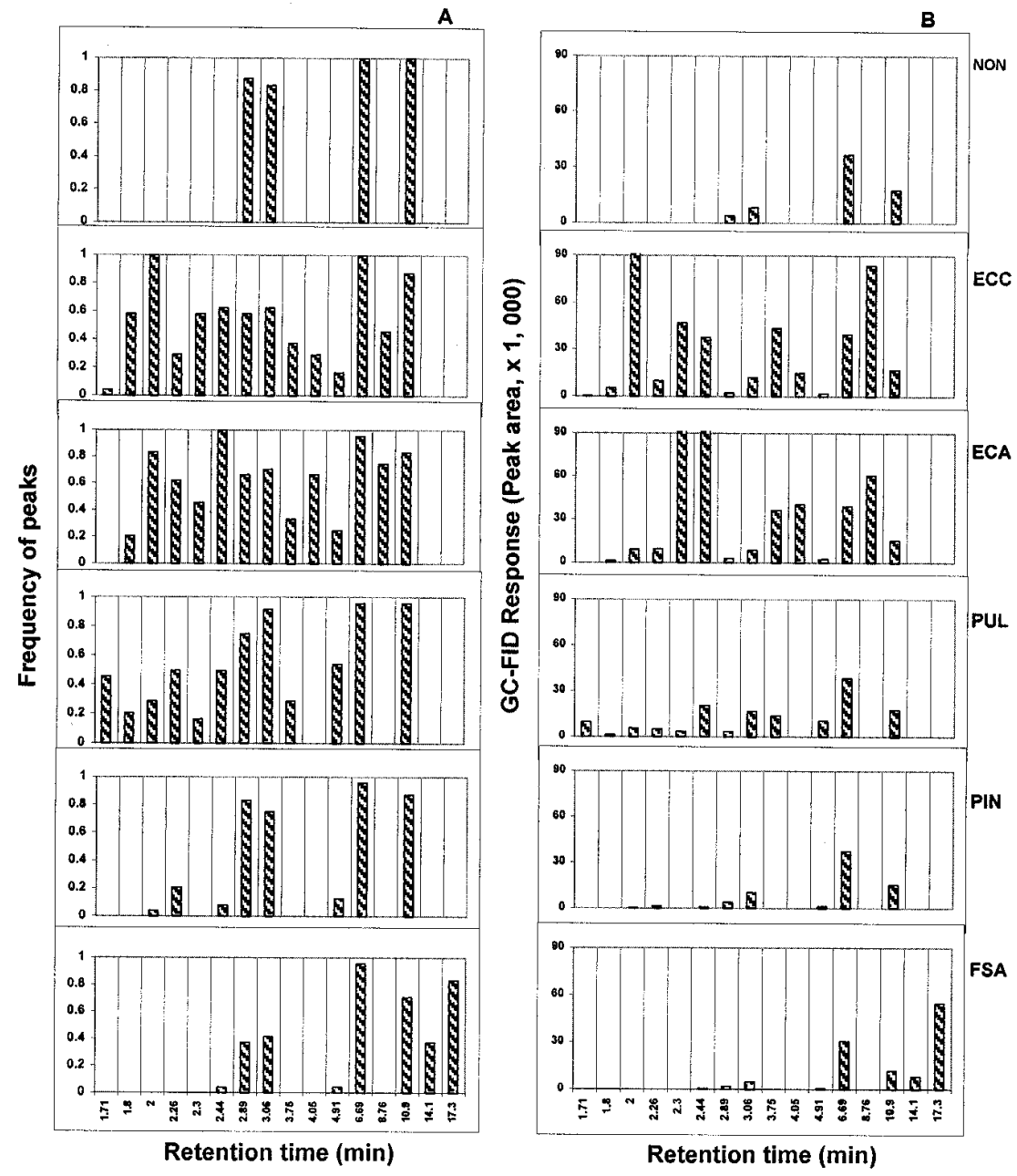

Fig. 3. A, Proportion of maximum ( 24 samples = three incubation times and eight blocks) frequency of volatile peaks (retention time [RT]) and B, average concentration of volatiles (peak area, for 24 samples) observed for six disease groups of potato: noninoculated (NON), or inoculated with Erwinia carotovora subsp. carotovora (ECC), E. carotovora subsp. atroseptica (ECA), Pythium ultimum (PUL), Phytophthora infestans (PIN), or Fusarium sambucinum (FSA), using solid phase microextraction traps and a gas chromatography. Volatiles were accumulated in bottles for $24 \mathrm{~h}$ and trapped with solid phase microextraction fiber for $15 \mathrm{~min}$. The RT for a given peak varied among treatments and blocks; therefore, a range of RTs was used to include a given RT. The 16 more consistent volatile peaks observed, average values (range), were: $\mathrm{RT}=1.71(1.69-1.72), 1.80(1.77-1.83)$, 2.0 (1.95-2.04), 2.26 (2.25-2.27), 2.3 (2.29-2.31), 2.44 (2.43-2.45), 2.89 (2.86-2.91), 3.06 (3.043.07), 3.75 (3.73-3.77), 4.05 (4.03-4.06), 4.91 (4.89-4.93), 6.69 (6.65-6.72), 8.76 (8.75-8.77), 10.93 (10.90-10.96), 14.11 (14.10-14.12), and $17.29(17.28-17.30)$.

groups and three incubation times) of profile prints. The network output consisted of maximum likelihood values (discriminant values) for each of the six disease groups, calculated for each observation fingerprint in the test file. An observation was classified into the disease group that had the highest predicted discriminant value. Percentages of correct classification of test observations were calculated for each of the eight network models, from which the percentage of correct classification of a given disease group was calculated, for both learn and cross-validation networks.

\section{RESULTS}

Volatile dynamics. The number of volatile compounds (RT) detected in a head space sample varied among disease groups, incubation time, and blocks. More than 22 different compounds were detected by the GC-FID analysis. Sixteen peaks that appeared in at least four blocks in any of the disease groups were selected as potential disease discriminators. Sample chromatogram fingerprints for the six disease groups obtained 5 days after inoculation are presented in Figure 2. The frequencies (proportion of maximum number of peaks) and the average concentration (peak area) of the 16 selected peaks for six diseasegroups, over three incubation times and eight blocks, are presented in Figure 3A and $\mathrm{B}$. The RT for a given peak varied up to 0.04 min over blocks; therefore, a range of RT values (only the RT mean value is referenced in the text) was used to assign a given RT. The occurrence of a given peak was not very consistent among blocks, and the frequency of occurrence depended on the peak. Only five peaks had a variation of $>0.02 \mathrm{~min}$. Identification of compounds, using GC/MS, is needed for clarification. Peak presence also depended upon incubation time; $F$. sambucinum disease-specific peaks were not present on tubers incubated for 3 days, in four out of eight blocks. $F$. sambucinum-specific peaks appeared quite consistently on 4 and 5 days of incubation, at which time there was visible dry rot symptoms. In tubers inoculated with $E$. carotovora subsp. carotovora, E. carotovora subsp. atroseptica, and Pythium ultimum, the lesions from different inoculation sites coalesced in 5 days of incubation, whereas those inoculated with $F$. sambucinum and Phytophthora infestans had only small lesions (4 to $8 \mathrm{~mm}$ in diameter). $P$. infestans produced fluffy mycelia. The NON potatoes developed no disease symptoms during the study. Tubers in general were apparently disease free, except for some that had a few scab lesions.

The concentration of volatiles produced varied with peaks and disease groups. Although some disease groups had similar peaks, the concentration varied differentially (Fig. 3B). For example, E. carotovora subsp. carotovora produced higher amounts of volatiles than E. carotovora 
subsp. atroseptica at RT $=2.0 \mathrm{~min}$ and lower amounts at RT $=2.3$ and $2.44 \mathrm{~min}$. Within a disease group, the concentration of a few volatiles, in a few blocks, increased with incubation time, which was associated with increased disease severity (for incubation time and blocks, data not shown). The variations among blocks also were quite high; therefore, the differences among incubation time were not dramatic.

Key criteria to discriminate disease groups. The volatile fingerprint, a pattern of peaks with specific RT, varied for different disease groups (Fig. 3A) and some peaks were quite specific to a given disease group. Even though the diseasespecific peaks didn't appear in all eight blocks of the experiment conducted here, it is possible to use a few selected peaks to discriminate disease groups. NON, Phytophthora infestans, and F. sambucinum produced very few compounds, whereas the rest (E. carotovora subsp. carotovora, E. carotovora subsp. atroseptica, and Pythium ultimum, ) produced many compounds, especially at short RTs. NON had four peaks (RT $=2.89,3.06,6.69$, and $10.93 \mathrm{~min}$ ), and $F$. sambucinum had distinct peaks at $\mathrm{RT}=14.1$ and $17.3 \mathrm{~min}$, in addition to those found for NON. Phytophthora infestans had a couple of peaks more than NON, but they were inconsistent and, thus, quite similar to NON. Pythium ultimum had a distinct peak at $\mathrm{RT}=1.71$ min and, in addition, could be discriminated from E. carotovora subsp. carotovora and E. carotovora subsp. atroseptica, which had distinct peaks at RT $=4.05$ and $8.76 \mathrm{~min}$. E. carotovora subsp. carotovora and E. carotovora subsp. atroseptica produced no clearly distinguishable peak and, therefore, could not be differentiated based on peaks alone. However, E. carotovora subsp. carotovora and E. carotovora subsp. atroseptica differed with respect to the amount of certain volatiles produced, especially at $\mathrm{RT}=2.0($ E. carotovora subsp. carotovora $=325,076$ and $E$. carotovora subsp. atroseptica $=9,194 \mathrm{GC}$ units) and RT $=2.44$ (E. carotovora subsp. carotovora $=37,701$ and $E$. carotovora subsp. atroseptica $=134,657$ GC units). For all disease groups, the peaks were not present all the time; thus, the lack of distinct peaks in some blocks for certain disease groups can lead to misclassification of fingerprints.

NNs to discriminate disease groups. The back-propagation network was developed to discriminate six disease groups. The network consisted of 16 processing elements as inputs (peak RT) and 6 as outputs (disease groups). A classification rate of $90 \%$ was obtained with 150,000 learning runs, after which the rate reduced (Fig. 4A). A maximum classification rate of $90 \%$ was obtained with 30 processing elements in two hidden layers, 20 in the first and 10 in the second (Fig. 4B). A maximum classification rate of $97 \%$ was obtained with a combination of Delta rule as a learning rule and TanH as a transfer function (Fig. 4C). A "learning" network was developed using a data set with all eight blocks and verified using the same data set used for learning. The learn network classified $100 \%$ of the profiles of $E$. carotovora subsp. carotovora, E. carotovora subsp. atroseptica, and Pythium ultimum and $95.8 \%$ of NON, Phytophthora

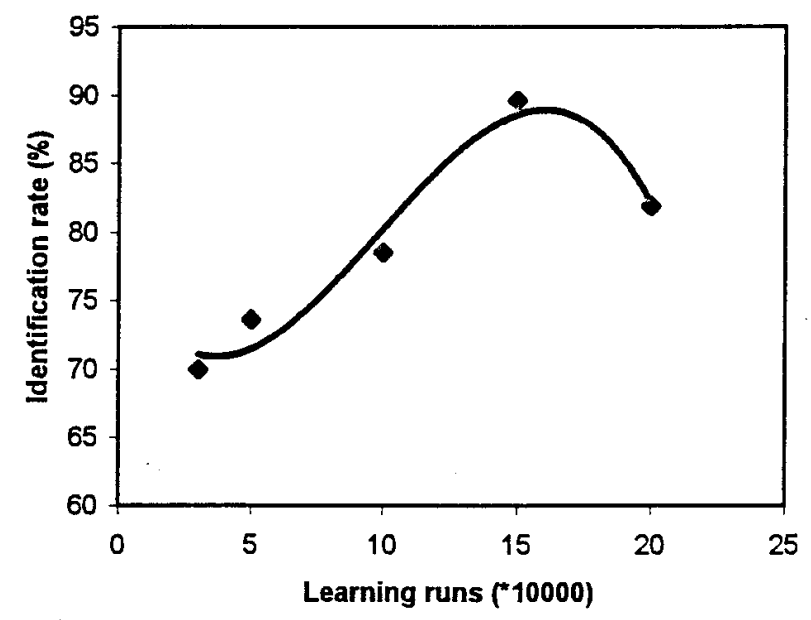

(A)

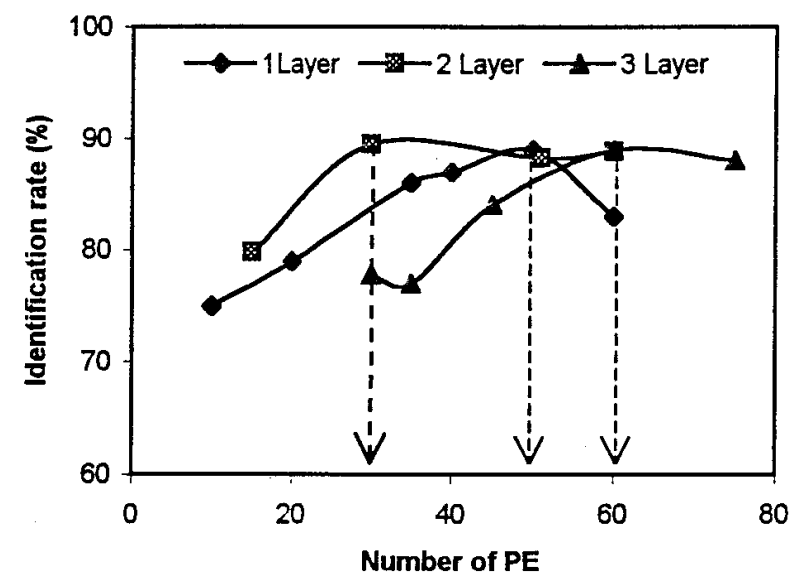

(B)

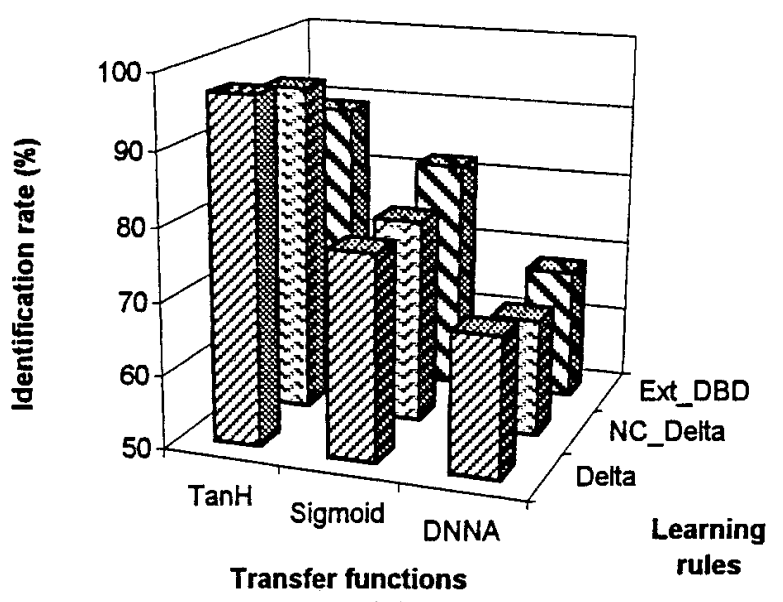

(C)

Fig. 4. Back-propagation network (of neural network) to discriminate six disease groups of potato, involving 16 processing elements (peaks or retention times) as input, and six elements (disease groups) as output. The main configuration parameters used for the optimization of classification rates were $\mathbf{A}$, effect of number of learning runs on the classification rate, $\mathbf{B}$, effect of number of hidden layers and number of processing elements (PE) in different layers on the classification rate, and $\mathbf{C}$, effect of combinations of transfer functions and learning rules on the classification rate. 
infestans, and $F$. sambucinum. The classification probability of profile prints to disease groups, based on cross-validation, varied among blocks from 50 to $94 \%$. The average probability of classification of unknown volatile fingerprints to disease groups based on cross-validation was as follows: $\mathrm{NON}=71 \%$, E. carotovora subsp. carotovora $=71 \%$, E. carotovora subsp. atroseptica $=71 \%$, Pythium ultimum $=$ $67 \%$, Phytophthora infestans $=46 \%$, and F. sambucinum $=75 \%$.

\section{DISCUSSION}

In this study, we have developed a method to detect and discriminate six disease groups of potato using SPME fibers and GC-FID. The method involved keeping tubers in closed containers for $24 \mathrm{~h}$ to accumulate volatiles, developing fingerprints using SPME and GC-FID, and discriminating disease groups on the basis of the qualitative criteria or the backpropagation networks. The qualitative criteria, based on RT, can be used to discriminate most disease groups. F. sambucinum can be separated based on one or two distinct peaks. Pythium ultimum can be separated from E. carotovora subsp. carotovora and E. carotovora subsp. atroseptica, though the latter two cannot be separated based on peaks alone. The relative amounts of volatiles produced by E. carotovora subsp. carotovora and $E$. carotovora subsp. atroseptica differentially varied with types of volatiles produced (in peaks at 2.0, 2.3, and $2.44 \mathrm{~min}$ ). Phytophthora infestans can be separated from all, except from NON. The efficiency of these criteria to discriminate diseases in practice, however, may be limited because not all combinations of peaks and variations in concentration of volatiles in certain peaks could be effectively accounted for in developing criteria. On the other hand, the network developed here considered complex relationships among fingerprints, rather than just a few peaks. It also provides an output on probability of classification of an unknown. The "potato disease NN" is robust and could be used as a practical tool to make disease management decisions. For friendly use of the network developed here, deployment networks can be developed to classify unknown profiles, which could be run using personal computers without the NN software.

The method developed here has the potential for commercial application for early detection and discrimination of diseases of potato tuber samples collected after harvest. However, it requires more studies involving other significant factors, more replicates, tests under commercial conditions, etc., before recommending it for commercial use. Important factors, other than those studied here, influence volatile dynamics. In our study, only five diseases were considered. The potato disease network developed here only discriminates diseases and does not necessarily identify them; therefore, an elaborate study considering most of the common tuber diseases is required to reduce missclassification of unknown samples to one of the disease groups tested here. In the present study, the tubers were surface sterilized to obtain fingerprints of individual pathogens, without alteration by saprophytes. We have studied only one disease at a time because outbreaks of a single disease are common in storage. However, the ability of the networks to discriminate diseases of nonsterilized tubers, in disease mixes, at different storage temperatures, and other conditions, should be explored. Some volatiles produced by a given tuber in the beginning disappeared with increase in incubation time. Volatiles react with each other and make different compounds, or volatiles can be metabolized by fruits and vegetables (7). Such reactions also may explain the variability in volatile profiles among blocks. Also, these models need to be tested with different cultivars, pathological and nonpathological causes of tuber deterioration, and other variables before recommending the use for commercial applications. In our study, the tubers for different blocks were bought over a period of 6 months from retail stores which received potatoes grown in different regions of Canada and the United States and stored for various lengths of time. These variabilities reflect on the robustness of the network developed.

Volatile production is related to active disease development. They are produced mainly as a result of enzymatic degradation of host tissue by pathogens. In 3 days of incubation time, $F$. sambucinum produced no disease-specific volatiles in $50 \%$ of the blocks, but did produce unique volatile signatures after 4 and 5 days of incubation. In 3 days of incubation, dry rot symptoms were not visible and became visible later. Still, the amount of tissue diseased was less than for other diseases, especially those with E. carotovora subsp. carotovora, E. carotovora subsp. atroseptica, and Pythium ultimum. Phytophthora infestans produced a limited amount of disease, though it produced a lot of mycelia. Different incubation times were considered here to involve the different disease severity which is normally observed under commercial conditions. However, commercial samples would need incubation at room temperature only if infection is not established or not active. Although, in this study, a 24-h volatile accumulation time was used, a preliminary study with $E$. carotovora subsp. carotovora produced many volatiles at $12 \mathrm{~h}$, making it possible to reduce the accumulation time.

We have used SPME fibers to trap volatiles because it takes less time to sample volatiles and the sensitivity is proven $(14,21)$. All the volatiles used were eluted within $20 \mathrm{~min}$ and, thus, it is possible to reduce the time required for $\mathrm{GC}$ analysis. The simplicity should make it more suitable commercially. Adsorption traps have been used in the past to trap volatiles and to detect diseases $(12,13,16,24,25)$. Loading and desorption of volatiles from adsorption traps take more time and extra equipment compared with SPME. Adsorption traps were found to trap, in general, more compounds and hold them at higher concentrations, but the GC-FID run time is longer than with SPME (14). Nonetheless, SPME traps have been used to sample flavor compounds from apple and were as efficient as adsorption traps (21). The quantitative response of the SPME trap was linear until saturation of the fiber, although the time taken to reach saturation varied with analytes, depending on their adsorption kinetics (21). For apple flavor compounds, most volatiles reached an equilibrium in $<12$ min (21) and microbial spoilage of milk was discriminated using a 12-min sampling time. In our study, the SPME fiber was retained in the headspace for $15 \mathrm{~min}$ to trap volatiles. Longer equilibrium time is required if concentration of compounds is critical. Some compounds did not reach an equilibrium even in $24 \mathrm{~min}$ (21). PDMS/Carboxen fibers are recommended for sampling compounds smaller than $\mathrm{C} 12$, and most compounds produced by E. carotovora subsp. carotovora in potato are within this range (5). Though the volatile fingerprints reported here for different diseases of potato are not comprehensive, they are quite discriminatory.

The network developed here was validated using the jackknife method, which can be considered the unbiased test for the validation of networks. The use of networks to assign an observation volatile profile to one of the potato disease groups is related to a "nose discriminant method," whereby a human nose classifies odors without really identifying specific compounds (14). However, the compound identity is quite useful to understand the disease-volatile dynamics, and for network stability. GC/MS has been used to identify compounds involved in peaks of GC output.

The techniques established here could be used to evaluate plant disease control options, such as host resistance, chemical, biological, and physical methods. The SPME-GC could be coupled with MS to identify the compounds; such information will help us better understand the mechanisms involved in disease suppression. The sampling method is nondestructive; therefore, the effect of control methods on disease progress over time can be monitored.

The method developed here to discriminate six potato disease groups can be used by storage managers to assess the quality of tuber samples collected at harvest or during storage. The presence of disease, type of disease, and infection incidence of 
tubers sampled can be assessed by incubating individual tubers, or a subsample of 2 to 5 tubers, in closed containers to produce volatiles. The method developed here can be used to detect the presence of a single disease with high probability. Although, in this study, tubers were incubated for at least 3 days for the disease to develop, it takes only $24 \mathrm{~h}$ to accumulate detectable volatiles in tubers with established infections coming from commercial storage, as compared with other methods that require a few weeks (22). Also, this method has the potential to detect diseases with similar symptoms (6). This knowledge base on disease potential could be used to make intelligent management decisions, such as storage of lots with higher disease potential at lower temperatures (10), the choice to market them sooner, and so on. The method must be scaled up to handle larger samples, such as a pallet box or room, for online application. The efficiency of SPME, as compared with adsorbent traps, to sample volatiles from big storage rooms where the concentration is in high parts per billion and variable spatially, is yet to be demonstrated $(13,24)$. Identification of volatile compounds using a mass spectrometer is necessary to develop a stable GC-FID-based system to discriminate diseases. Electronic noses with an array of many (6 to 32) sensors have been used to detect deterioration of various food products and to identify pathogens $(8,9)$, but their sensitivity, resolution, and use to identify compounds are limited compared with GC-FID or GC/MS. Portable GC-FID equipment, already available commercially, suggests potential alternatives to make this technology more feasible under commercial conditions.

\section{ACKNOWLEDGMENTS}

We thank CORPAQ and NSERC-RG for financial support; M. Lacroix for providing the cultures of Erwinia carotovora subsp. carotovora, E. carotovora subsp. atroseptica, Pythium ultimum. and Fusarium sambucinum; and H. Platt for cultures of Phytophthora infestans.

\section{LITERATURE CITED}

1. Ayers, W. A., and Lumsden, R. D. 1975. Factors affecting production and germination of oospores of three Pythium spp. Phytopathology 65:1094-1100.

2. Bartz, J. A., and Eckert, J. W. 1987. Bacterial diseases of vegetable crops after harvest. Pages 351-375 in: Postharvest Physiology of Vegetables. J. Weichmann, ed. Marcel Dekker Inc., NY.

3. Batchelor, W. D., Yang, X. B., and Tschanz, A. T. 1997. Development of a neural network for soybean rust epidemics. Trans. ASAE 40:247-252.

4. De Boer, S. H., De Haan, T. L., and Mawhinney, J. 1989. Predictive value of post harvest serological tests for bacterial ring rot of potato. Can. J. Plant Pathol. 11:317-321.

5. De Lacy Costello, B. P. J., Evans, P., Ewen, R. J., Gunson, H. E., Ratcliffe, N. M., and Spencer-Phillips, P. T. N. 1999. Identification of volatiles generated by potato tubers (Solanum tuberosum cv: Maris Piper) infected by Erwinia carotovora, Bacillus polymyxa and Artrobacter sp. Plant Pathol. 48:345-351.

6. De Lapeyre de Bellaire, L., Chillet, M., and Mourichon, X. 2000. Elaboration of an early quantification method of quiescent infections of Colletotrichum musae on bananas. Plant Dis. 84:128-133.

7. Hamilton-Kemp, T. R., Archbold, D. D., Loughrin, J. H., Collins, R. W., and Byers, M. E. 1996. Metabolism of natural volatile compounds by strawberry fruit. J. Agric. Food Chem. 44:2802-2805.

8. Jonsson, A, Winquist, F., Schnürer, J., Sundgren, H., and Lundström, I. 1997. Electronic nose for microbial quality classification of grains. Int. J. Food Microbiol. 35:187-193.

9. Keshri, G., Magan, N., and Voysey, P. 1998. Use of E-nose for early detection and differentiation between spoilage fungi. Lett. Appl. Microbiol. 27:261-264.

10. Kushalappa, A. C., and Zulfiqar, M. Effect of wet incubation time and temperature on infection, and of storage time and temperature on soft rot lesion expansion in potatoes inoculated with Erwinia carotovora ssp. carotovora. Potato Res. In press.

11. Lacroix, R., Salehi, F., Yang, X. Z., and Wade, K. M. 1997. Effects of data processing on the performance of artificial neural networks for dairy yield prediction and cow culling classification. Trans. ASAE 40:839-846.

12. Lougheed, E. C., Murr, D. P., and Toivonen, P. M. A. 1987. Ethylene and nonethylene volatiles. Pages 255-76 in: Postharvest Physiology of Vegetables. J. Weichmann, ed. Marcel Dekker, Inc., NY.
13. Lyew, D., Gariepy, Y., Ratti, C., Raghavan, G. S. V., and Kushalappa, A. C. 1999. An apparatus to sample volatiles in a commercial potato storage facility. Appl. Eng. Agric. 15:243-247.

14. Marsili, R. T. 1999. SPME-MS-MVA as an electronic nose for the study of off-flavor in milk. J. Agric. Food Chem. 47:648-654.

15. NeuralWare. 1993. NeuralWorks: Advanced Referenced Reference Guide for NeuralWorks Professional II/PLUS and NeuralWorks Explorer. NeuralWare, Inc., Carnegie, PA.

16. Ouellette, E., Raghavan, G. S. V., Reeleder, R. D., and Greenhalgh, R. 1990. Volatile profiles for disease detection in stored potatoes. J. Food Process. Preserv. 14:279-300.

17. Peters, R. D., Platt, H. W., Hall, R., and Medina, M. 1999. Variation in aggressiveness of Canadian isolates of Phytophthora infestans as indicated by their relative abilities to cause potato tuber rot. Plant Dis. 83:652-661.

18. Ranganna, B., Kushalappa, A. C., and Raghavan, G. S. V. 1997. Ultraviolet irradiance to control dry rot and soft rot of potato in storage. Can. J. Plant Pathol. 19:30-35.

19. Rowe, R. C., ed. 1993. Potato Health Management. American Phytopathological Society Press, St. Paul, MN.

20. Schnürer, J., Olsson, J., and Börjesson, T. 1999. Fungal volatiles as indicators of food and feed spoilage (review). Fungal Genet. Biol. 27:209-217.

21. Song, J., Gardner, B., Holland, J. F., and Beaudry, R. M. 1997. Rapid analysis of volatile flavor compounds in apple fruits using SPME and GC/time-of-flight mass spectrometry. J. Agric. Food Chem. 45:1801-1807.

22. Theron, D. J., 1991. Prediction of potato dry rot based on the presence of Fusarium in soil adhering to tubers at harvest. Plant Dis. 75:126-130.

23. Toivonen, P. M. A., 1997. Non-ethylene, nonrespiratory volatiles in harvested fruits and vegetables: their occurrence, illogical activity and control. Postharvest Biol. Technol. 12:109-125.

24. Varns, J. L., and Glynn, M. T. 1979. Detection of disease in stored potatoes by volatile monitoring. Am. Potato J. 56:185-197

25. Waterer, D. R., and Pritchard, M. K. 1984. Volatile monitoring as a technique for differentiating between $E$. carotovora and $C$. sepedonicum infections in stored potatoes. Am. Potato J. 61:345-353.

26. Waterer, D. R., and Pritchard, M. K. 1985. Production of volatile metabolites in potatoes infected by Erwinia carotovora var. carotovora and E. carotovora var. atroseptica. Can. J. Plant Pathol. 7:47-51. 